\title{
IMPROVEMENT OF THE ACCURACY OF THE ORIENTATION OF AN OBJECT BY USING A PAIR OF ROTATING IMS
}

\author{
Pavol KAJÁNEK ${ }^{*}$, Alojz KOPÁČIK ${ }^{1}$
}

\section{Abstract}

Inertial measurement systems (IMS) belong among navigation systems which permit the monitoring of the position and orientation of an object in three-dimensional space. The functional principle of IMS is based on the integration of the output signal (acceleration and angular rate) of inertial sensors to the actual position and orientation of IMS. This integration causes the rapid accumulation of systematic IMS's errors, which leads to a rapid increase in position and orientation errors over time.

The paper deals with the design of a prototype for a pair of lowcost IMSs, which are regularly rotated around their vertical axis by stepper motors. To eliminate the systematic errors of gyroscopes in the direction of the rotation axis, two counter-rotating IMSs are used. An optimal model of the data processing was developed and presented.
Address

1 Department of Surveying, Slovak University of Technology, Bratislava, Slovakia

* Corresponding author: pavol.kajanek@stuba.sk

\section{Key words}

- Controlled rotation,

- IMS,

- Gyroscope,

- Systematic error

- Elimination

- Base.

\section{INTRODUCTION}

An inertial measurement system (IMS) is a navigation system that consists of a group of inertial sensors and a navigational computer placed on a common platform. An IMS allows for the continual monitoring of an object's movements. Thanks to the high frequency of their acquisition of data, they are appropriate for monitoring highly dynamic processes. An IMS is independent of an external signal, which allows it to be used in environments where other systems fail (the indoor environment of buildings, underwater, tunnels and forests).

The principle of determining a position when using an IMS (Fig. 1 ) is based on a recursive algorithm and is usually presented as a dead reckoning. The actual position $P(t+1)$ is calculated from a previous position $P(t)$, the distance travelled $d(t)$, and the orientation of this movements . The position of the IMS is defined in a reference coordinate system $\left(\mathrm{CS}^{\mathrm{R}}\right)$. The coordinate system of an IMS CS ${ }^{\mathrm{IMS}}$ is defined by the orientation of the sensitive axes of the inertial sensors.

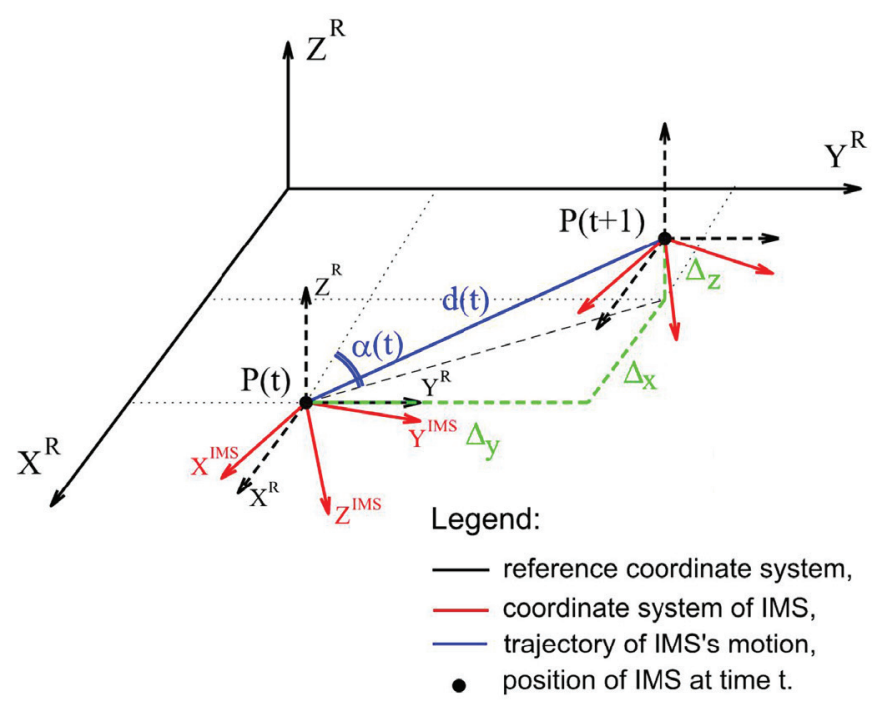

Fig. 1 Principle of position determination using IMS 


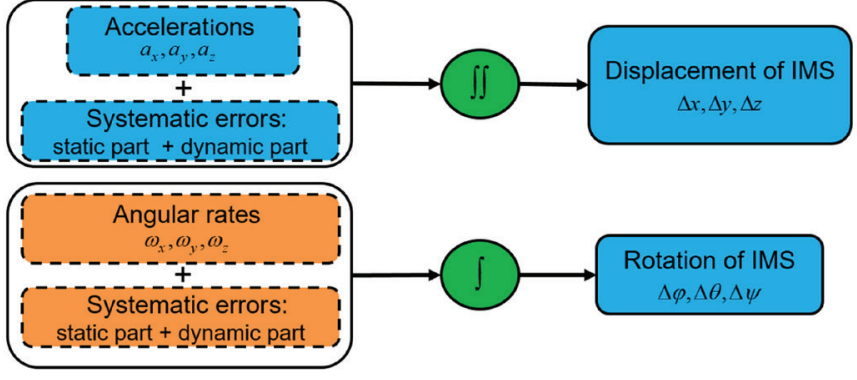

Fig. 2 Accumulation of systematic errors of an IMS signal in the integration process

The integration of inertial measurements (accelerations, angular rates) to the translation and rotation of an IMS's platform leads to the rapid accumulation of systematic errors of the inertial measurements in their actual position and orientation (Groves, 2008) (Fig. 2).

Systematic errors of inertial measurements consist of static and dynamic parts. The static part of a systematic error (SPSE) can be eliminated by using the error's model of the inertial sensors, which was defined by the calibration of the sensor used. The dynamic part of the systematic error (DPSE) cannot be eliminated by calibration, because any changes during measurement depends on the dynamics of the IMS's movement. The presence of a DPSE in the IMS's signals leads to an increase in the position and orientation errors along with the increasing time of measurement (Fig. 3). This effect is multiplied by increasing the frequency rate.

A zero velocity update (ZUPT) is often used to eliminate systematic errors of an accelerometer. The principle of this method is based on the condition of the zero velocity at a time when the IMS is stable. Based on this condition, the error's component of the calculated velocity is modelled as a linear function defined (calculated by the integration of an acceleration) at the moment of starting and stopping the IMS.

The ZUPT allows for the elimination of only a linear part of the error's components of the velocity (Fig. 4). The nonlinear part of the error's components of the velocity generated by a change in the dynamics of the IMS's movement is accumulated into distance errors, which depend on the length of the time interval between the stops of the IMS.

The increased position error resulting from the insufficient elimination of the DPSE of the accelerometers with respect to the recur-
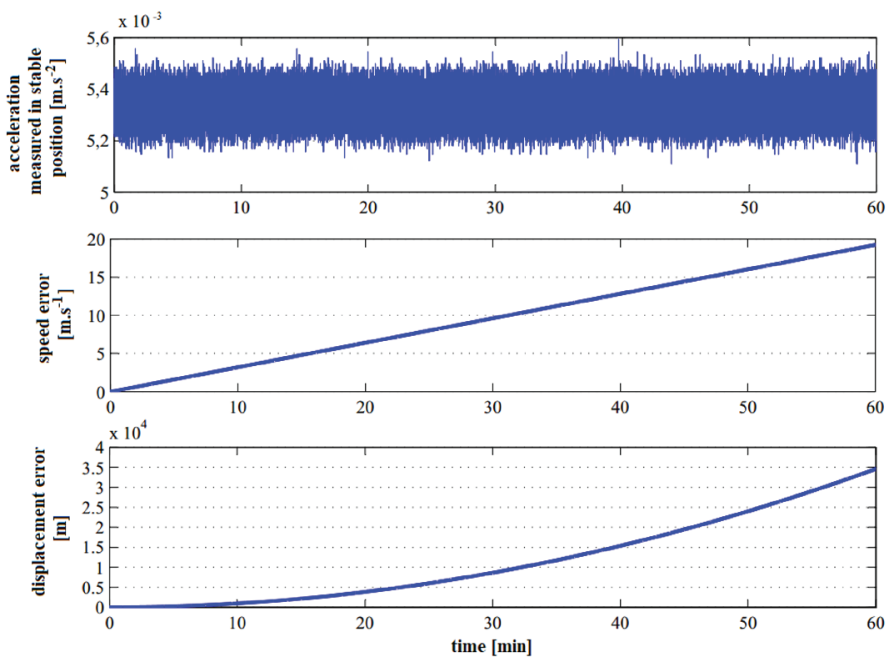

Fig. 3 Increase in the velocity and displacement errors generated by the double integration of the acceleration measured (the time series represents one hour of the measurement logging with a frequency rate of $100 \mathrm{~Hz}$ ) sive algorithm for determining a position. The solution most often used is a combination of an IMS with an additional sensor, which allows for the calculation of the error's component (as opposed to a ZUPT, in which it uses measurements/conditions only at a time when the IMS stops). The low-cost and simple application of an optical encoder lead to their frequent usage in IMS data processing (Yi, 2007; Wu, 2014; Ndjeng, 2011). Other approaches use ultrasound sensors (Xu, 2014), radio-frequency sensors RFID (Bekkali, 2007), GNSS (Reimer, 2017) or pseudo-satellites (Rizos, 2008), which are used with IMS for position corrections. But these solutions need the creation of an infrastructure of a transmitter with a known position, which is a time-consuming and expensive process.

\section{ELIMINATION OF THE SYSTEMATIC ERRORS OF INERTIAL SENSORS USING THE CONTROLLED ROTATION OF IMS}

A new approach for eliminating the systematic errors of inertial sensors is based on using the rotational motion of the IMS's platform (Ben, 2011; Collin, 2015; Sun, 2012). An rotating inertial measurement system (RIMS) generates periodical changes in the orientation of the sensitive axis of an inertial sensors, which leads to periodical changes in the DPSE with a zero mean value (after eliminating centrifugal acceleration generated by the rotation). The periodic change of the value (and sign) of DPSE leads to its elimination within each rotational period.

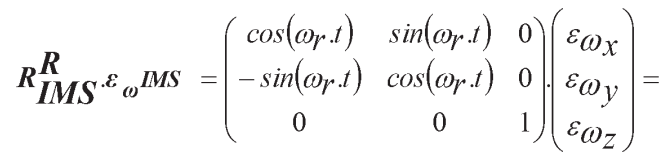

$$
\begin{aligned}
& =\left(\begin{array}{c}
\varepsilon_{\omega_{x}} \cdot \cos \left(\omega_{r} \cdot t\right)+\varepsilon_{\omega_{y}} \cdot \sin \left(\omega_{r} \cdot t\right) \\
-\varepsilon_{\omega_{x}} \cdot \sin \left(\omega_{r} \cdot t\right)+\varepsilon_{\omega_{y}} \cdot \cos \left(\omega_{r} \cdot t\right) \\
\varepsilon_{\omega_{z}}
\end{array}\right) \\
& \boldsymbol{R}_{\boldsymbol{I M}}^{\boldsymbol{R}} \boldsymbol{\varepsilon}_{\boldsymbol{a}}^{\boldsymbol{I M S}}=\left(\begin{array}{ccc}
\cos \left(\omega_{r} . t\right) & \sin \left(\omega_{r} . t\right) & 0 \\
-\sin \left(\omega_{r} . t\right) & \cos \left(\omega_{r} . t\right) & 0 \\
0 & 0 & 1
\end{array}\right) \cdot\left(\begin{array}{l}
\varepsilon_{a_{x}} \\
\varepsilon_{a_{y}} \\
\varepsilon_{a_{z}}
\end{array}\right)= \\
& =\left(\begin{array}{c}
\varepsilon_{a_{x}} \cdot \cos \left(\omega_{r} . t\right)+\varepsilon_{a_{y}} \cdot \sin \left(\omega_{r} . t\right) \\
-\varepsilon_{a_{x}} \cdot \sin \left(\omega_{r} \cdot t\right)+\varepsilon_{a_{y}} \cdot \cos \left(\omega_{r} \cdot t\right) \\
\varepsilon_{a_{z}}
\end{array}\right)
\end{aligned}
$$

where:

$\omega_{r} \quad-$ angular rate of the controlled rotation of IMS,

$\varepsilon_{\alpha} I M S, \varepsilon_{\omega} I M S$ - systematic error of the acceleration/angular rate.

The periodical change of DPSE during the rotation of IMS is defined by a rotation matrix $\boldsymbol{R}_{I M S}^{R}$, which represents the rotation of the coordinate system of IMS $C S^{I M S}$ with respect to the reference coordinate system $\left(C S^{R}\right)$.

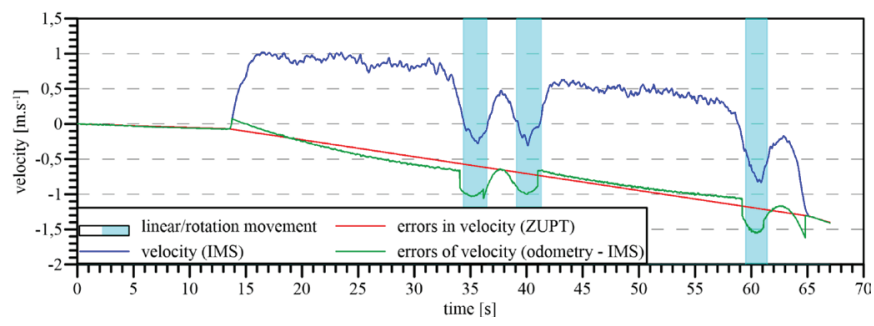

Fig. 4 Changes in the error's component of the calculated velocity affected by changes of dynamic state of the IMS 


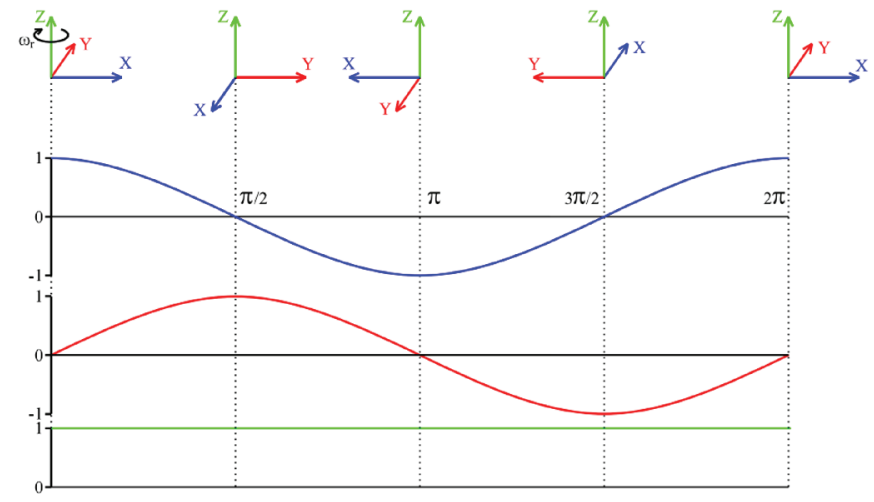

Fig. 5 Effect of the controlled rotation on the constant part of the systematic errors of RIMS during one period of rotation in the direction of a $Z$ axis

When a RIMS rotates around its $\mathrm{Z}$ axis, errors in the direction of the $\mathrm{X}, \mathrm{Y}$ axes are eliminated within each rotation period. The effect of the controlled rotation on the systematic errors of the inertial sensors (Fig. 5) can be expressed by using a coefficient in a range from -1.0 to 1.0 (the extreme values are obtained in cases when the sensor's axis is parallel to the axis of the reference coordinate system). This coefficient expresses a part of the total error represented in the actual measurement.

The controlled rotation of the RIMS allows for the elimination of the DPSE of sensors, the sensitive axis of which is perpendicular to the rotation axis of the RIMS. When a RIMS rotates around its $\mathrm{Z}$ axis (or the axis perpendicular to the plane of the IMS's movement), the DPSE of a sensor with a sensitive axis parallel to the RIMS rotation axis, could not be eliminated, which leads to increasing errors in the system's orientation.

\section{DESIGN OF THE DATA PROCESSING MODEL}

The proposed data processing model (Fig. 6) combines several procedures for eliminating systematic errors, namely:
- Application of the error models of the sensors used (SPSE elimination).

- Controlled rotation of the IMS (elimination of DPSE from inertial measurements in the direction of the axis perpendicular to the axis of the rotation).

- Using a pair of counter-rotating RIMS to eliminate the DPSE of the gyroscope's measurement in the direction of the axis parallel to the rotation axis.

Processing data of the inertial measurements made by RIMS is different from processing the measurements from an IMS with a stable platform, which is described in (Kopáčik, 2016). With regard to the controlled rotation, the orientation of the $\mathrm{CS}^{\mathrm{IMS}}$ continually changes with respect to $\mathrm{CS}^{\mathrm{R}}$. Therefore, the model for processing the data was completed by transforming the measurements realized in $\mathrm{CS}^{\mathrm{IMS}}$ into the $\mathrm{CS}^{\mathrm{R}}$, where the rotational angle was calculated from the angular velocity of the RIMS rotation.

In the next step, it is necessary to eliminate any centrifugal accelerations (generated by the controlled rotation) from the measured accelerations by the use of a Butterworth bandpass filter. The selection of this filter was conditioned by its maximally flat frequency response in the passband. The frequency range of the filter is defined based on the angular rate of the IMS platform's rotation $\omega_{\text {rot }}$ (Fig. 7):

$$
f_{\text {rot }}=\frac{\omega_{\text {rot }}}{2 \pi} .
$$

\section{1 Elimination of the systematic errors of a gyroscope with a sensitive axis parallel to a rotation axis}

As was previously mentioned, the RIMS does not eliminate the systematic errors of a sensor, the sensitive axis of which is parallel to a rotation axis. During the rotation of the IMS around the $\mathrm{Z}$ axis (or an axis perpendicular to the plane of the RIMS movements), the systematic errors of gyroscopes accumulate, which leads to increased orientation errors of the RIMS's movements. To eliminate the DPSE of the gyroscopes in the direction of the rotation axis, a pair of counter-rotating IMS platforms is used. Using a pair of counter-rotating

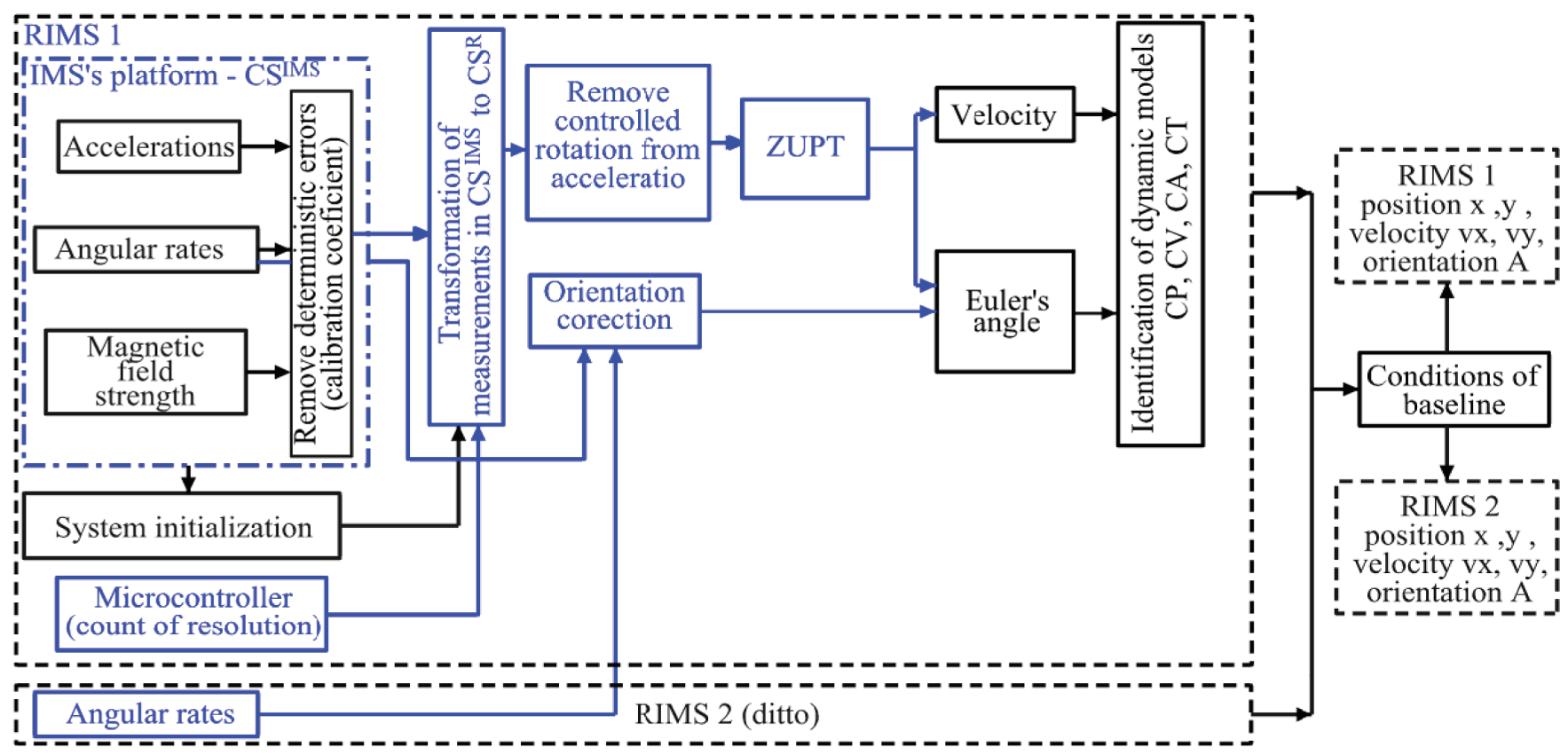

Fig. 6 Scheme of the proposed model 
a)

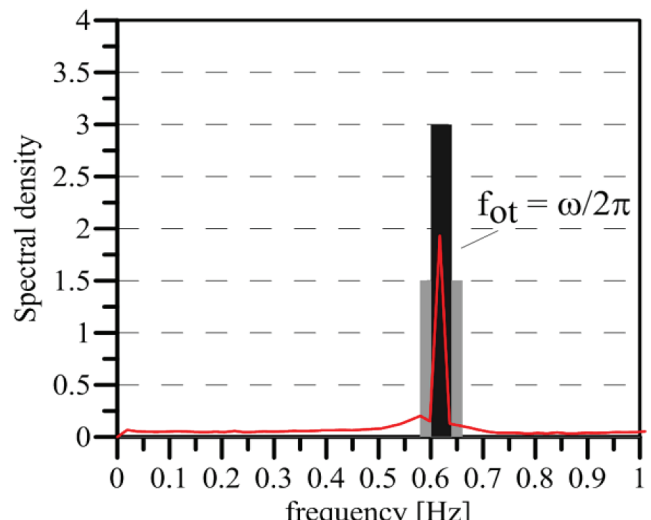

b)

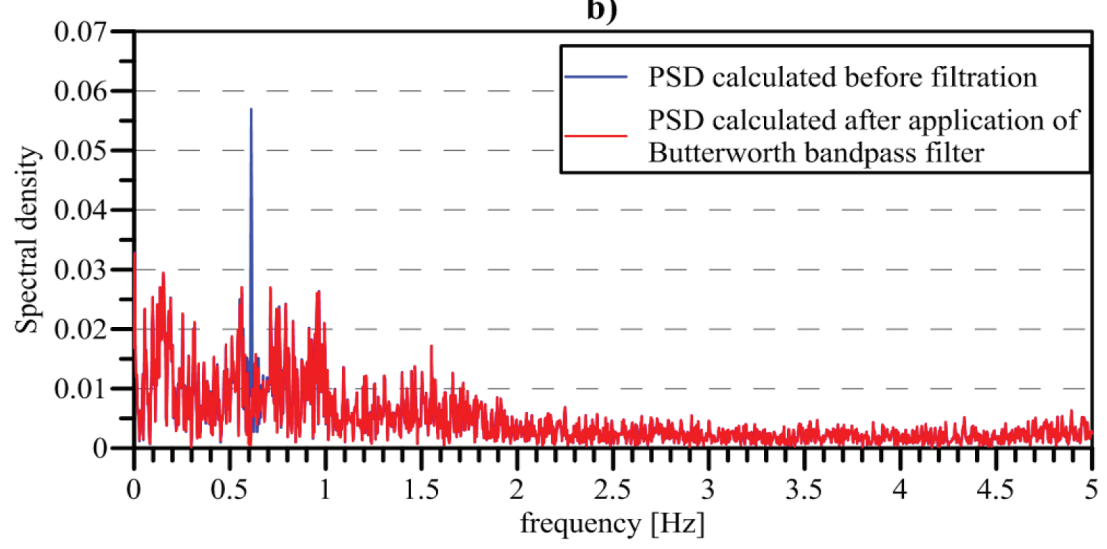

PSD from accelerations correspond to controlled rotation transition region stopband passband

Fig. 7 Elimination of centrifugal accelerations from the measured signal using a Butterworth bandpass filter (left - frequency response of the designed filter, right - power spectral density (PSD) of signal before and after using the filter)

a)

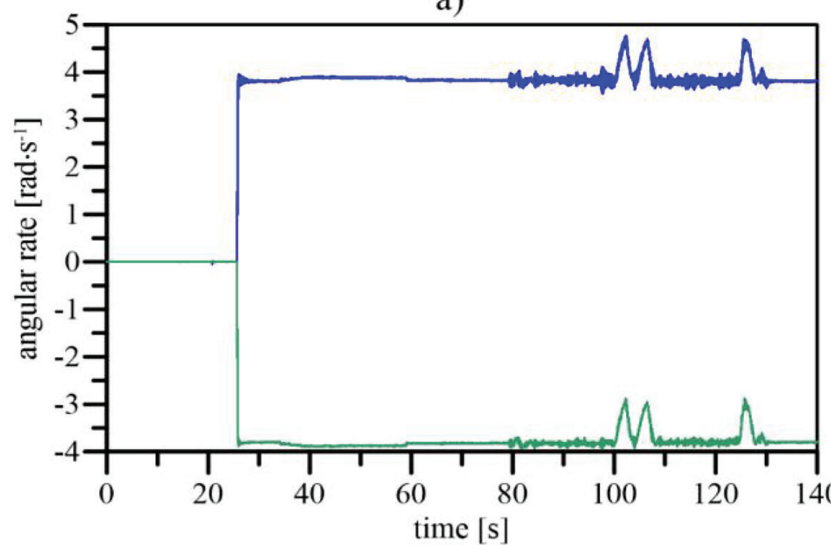

b)

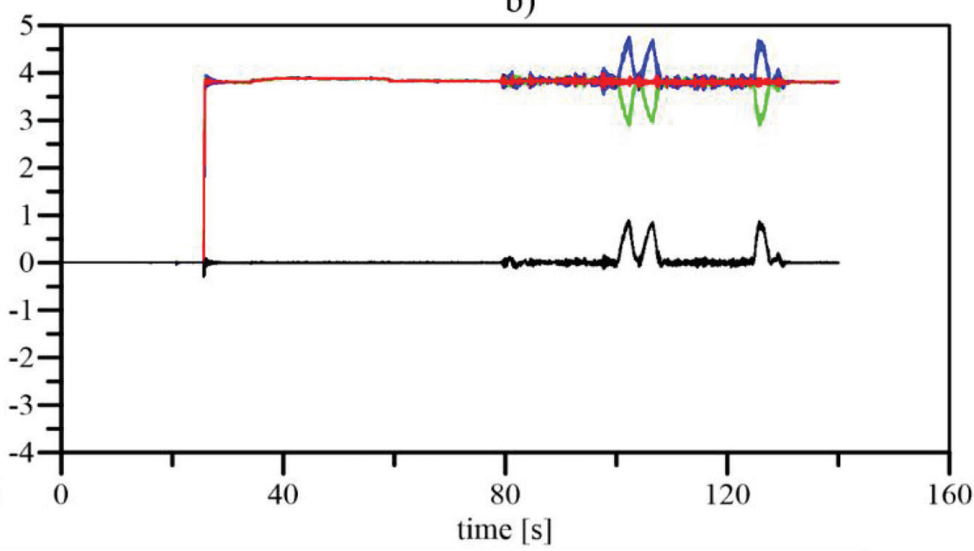

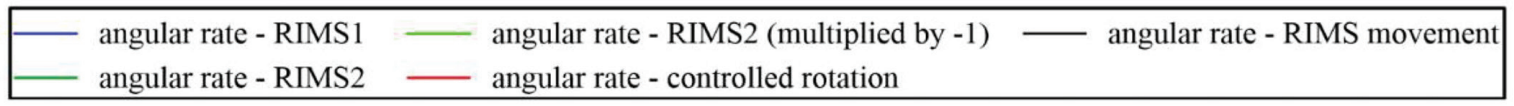

Fig. 8 Elimination of the DPSE of a gyroscope in the direction of the Z axis generated by the controlled rotation of RIMS (a-measured angular rate, $b$ - separation of the angular rate on component corresponding to controlled rotation and movement of measured system)

IMS generates the same dynamic state of both RIMS (due to the same angular rate of rotation), which influence the course of the DPSE.

The arithmetic mean of the angular velocities of both RIMS $\omega Z^{1}$, $\omega Z^{2}$ (formula 4 ) expresses an angular velocity corresponding to the controlled rotation of the RIMS's platform with the DPSE of the angular rate (Fig. $8 \mathrm{~b}$ - the red line). In calculating the arithmetic mean, the angular velocity of the second RIMS $\omega Z^{2}$ is explicitly assigned the opposite sign.

The angular rate $\omega_{m o v}$ corresponding to the movement of the measuring system (Fig. 8b - black line) is calculated by subtracting the angular velocity of the controlled rotation $\omega_{\text {rot }}$ from the angular rate $\omega Z^{1}$ measured by the RIMS:

$$
\begin{gathered}
\omega_{r o t}+\varepsilon_{\omega}=\left(\omega Z^{1}+\left(-\omega Z^{2}\right)\right) / 2, \\
\omega_{\text {mov }}=\omega Z^{1}-\omega_{r o t}+\varepsilon_{\omega} .
\end{gathered}
$$

\section{DESIGN OF MEASUREMENT SYSTEM}

In order to test the proposed model, a measuring system was designed. The construction of the measuring system consists of a trolley with a baseline. At both ends of the baseline an IMS was placed. Because of the installation of these two IMSs at the common baseline, a strong geometrical relation (constraint) was created between the reference systems of both IMSs. The distance between the centres of the reference systems of the RIMSs is constant with the value of $0.890 \mathrm{~m}$ (Fig. 9). The initial orientation of the coordinate systems of both RIMSs is defined at the beginning of measurement. The coordinate systems of both RIMSs are parallel, with their X axes oriented in the direction of the trolley's movement, the $Y$ axes parallel with the longitudinal axis of the baseline, and the $\mathrm{Z}$ axes perpendicular to the $\mathrm{XY}$ plane.

The controlled counter-rotating movement of the RIMSs with the same angular rate was realized by a pair of stepper motors, which are controlled by a stepper motor driver and same microcontroller. The measuring system was supplemented by an optical rotary encoder, which served to analyse the DPSE of the accelerometers. 


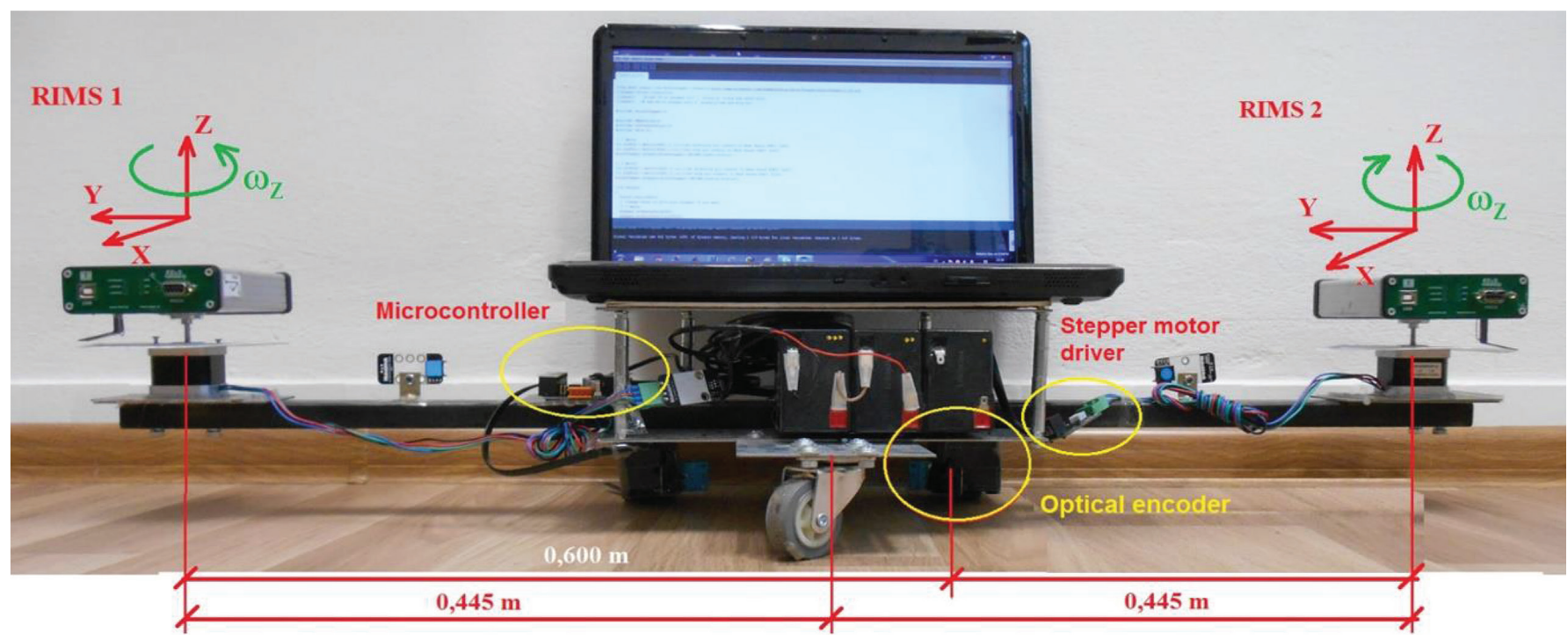

Fig. 9 Design of the measurement system

\section{EXPERIMENTAL MEASUREMENT AND VERIFICATION OF THE PROPOSED MODEL}

During the experimental measurements, the measurement system was moved along a predefined trajectory with a rectangular shape. The trajectory consists of straight lines; the transition between the straight lines of the trajectory was realized by an arc. The 9 control points $(\mathrm{CP})$ of the trajectory were located at the intersection of the straight lines with the arcs. These points were used to analyse the efficiency of the proposed model for eliminating the DPSE of the inertial sensors.

The resulting error in the position consists of two parts - an error in the determination of the trajectory's length (generated by the DPSE of the accelerometers) and the error in the orientation of the IMS's (generated by the DPSE of the gyroscopes). The elimination of the accelerometer's DPSE was analysed based on difference in distance, which was calculated as average value from differences calculated between reference and measured distances between the CPs. In the case of the gyroscopes, the model's efficiency was analysed based on the orientation drift, which was calculated as ratio between difference in the orientation of the straight part of the trajectory and time of measurement. The accuracy of the positioning $\Delta \delta$ was defined as the ratio between the difference in the position of the selected CP $\Delta p$ and the corresponding length of the trajectory travelled $d_{T T}$ :

$$
\Delta \delta=\frac{\Delta p}{d T T}
$$

According to the experimental results (Fig. 10), it could be concluded that the ZUPT only eliminates the linear part of the accelerometer's DPSE, which leads to an increasing error in the distance measured with the increased length of the time interval between the IMS stops (the average difference achieved during the experiment was up to $0.7 \mathrm{~m}$ ).

Using the RIMS to eliminate the DPSE from the accelerations measured results in a difference in the average accuracy of positioning of 0.009 , which corresponds to a difference in position $9 \mathrm{~mm} / 1 \mathrm{~m}$ of the trajectory travelled. This is comparable with the combination of the IMS and optical encoder, where the average accuracy of positioning is 0.005 . Therefore, the use of RIMS can be understood as an alternative solution in cases when an optical encoder cannot be used.

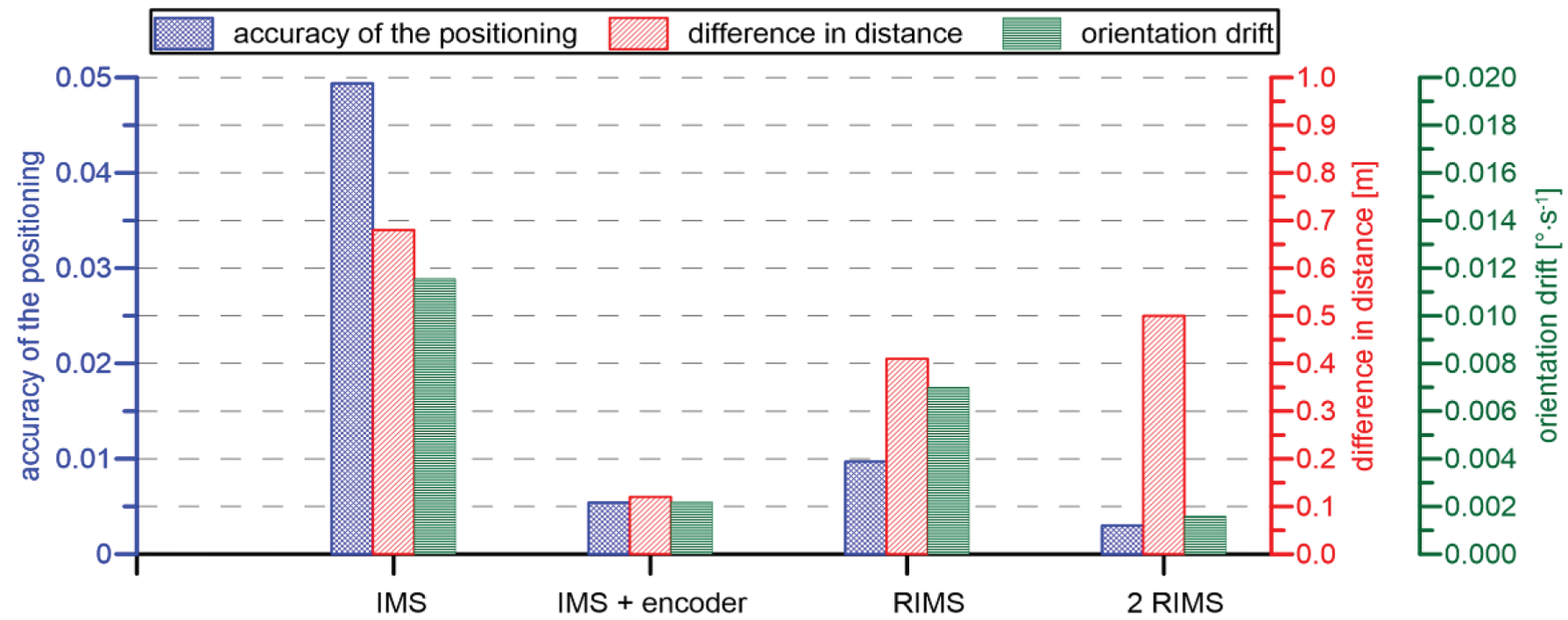

Fig. 10 Comparison of the effectiveness of the different approaches for eliminating systematic errors in the IMS's signal 
Tab. 1 Results obtained by using each individual model

\begin{tabular}{l|c|c|c}
\hline Model & $\begin{array}{c}\text { Accuracy of the } \\
\text { positioning }\end{array}$ & $\begin{array}{c}\text { Difference in } \\
\text { distance }[\mathrm{m}]\end{array}$ & $\begin{array}{c}\text { Orientation } \\
\text { drift }\left[{ }^{\circ} \cdot \mathrm{s}^{-1}\right]\end{array}$ \\
\hline IMS & 0.049 & 0.68 & 0.0115 \\
\hline IMS + encoder & 0.005 & 0.12 & 0.0021 \\
\hline RIMS & 0.009 & 0.41 & 0.0070 \\
\hline $\begin{array}{l}\text { Pair of counter } \\
\text { rotating RIMS }\end{array}$ & 0.003 & 0.50 & 0.0015 \\
\hline
\end{tabular}

Increasing the angular rate of the RIMS leads to a more effective elimination of the DPSE of the inertial sensors, because the period of the rotation's movement is shortened. During short periods, it can be assumed that the DPSE will not significantly change.

Larger differences in the distances calculated by the model and the RIMS are affected by the slow adaptation of the frequency filter that is applied to remove the centrifugal acceleration corresponding to the rotation movement of the RIMS. Changes in the angular rate of the RIMS's rotation negatively affect the accuracy of the position calculated.

The use of a pair of counter-rotating RIMS allows for the efficient eliminating of the DPSE of gyroscopes in the direction of the axis of rotation, which significantly increases the accuracy of the orientation of the RIMS movement (the orientation drift is $0.0015^{\circ} \cdot \mathrm{s}^{-1}$ ) compared to the use of one RIMS (the orientation drift is $0.0065^{\circ} \cdot \mathrm{s}^{-1}$ ).

The efficiency of the proposed model can be seen in the trajectory calculated using the proposed model (Fig. 13). The first trajectory was calculated using ZUPT (Fig. 11). ZUPT only eliminates the linear part of error's component of velocity, which leads to increasing the position errors of the time of measurement. The RIMS effectively eliminates the systematic errors of the accelerometer in the movement's direction, but cannot eliminate the systematic errors in the gyroscope's signal, which leads to increasing errors in the orientation (Fig. 12). The model based on the usage of a pair of rotating counter IMSs eliminate this problem effectively, which leads to the increased accuracy of both the orientation and the position of the IMS motion (Fig. 13).

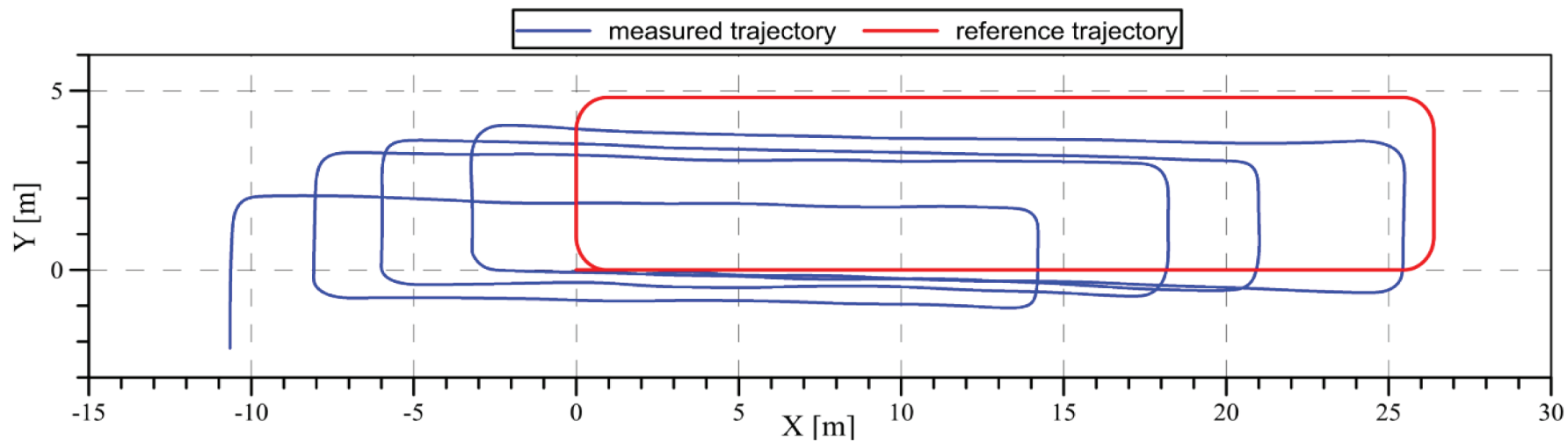

Fig. 11 Trajectory measured only by IMS using ZUPT

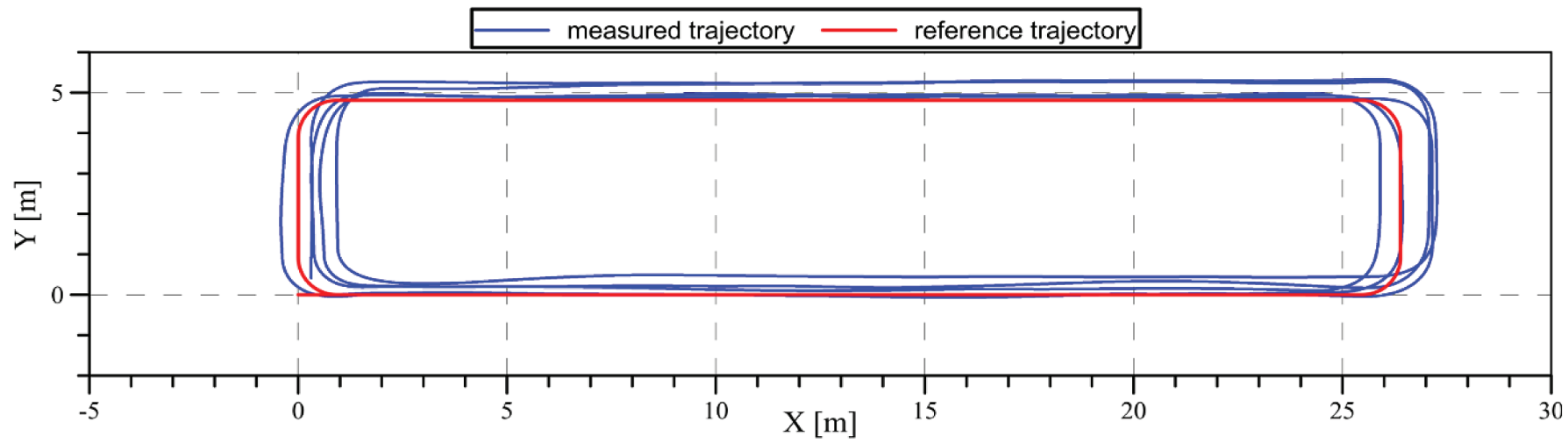

Fig. 12 Trajectory measured using RIMS

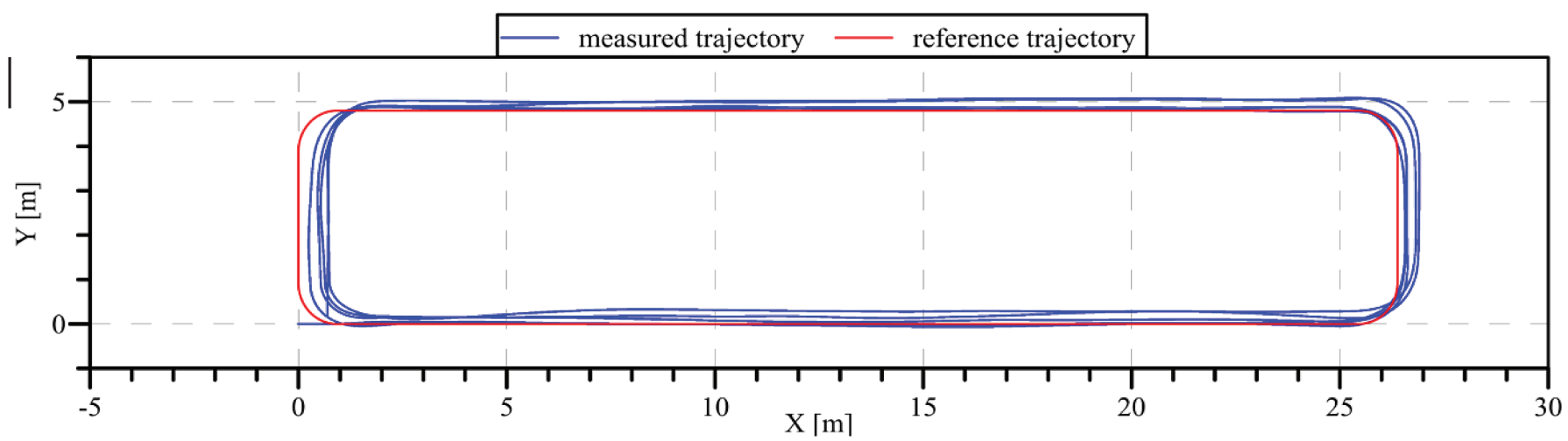

Fig. 13 Trajectory measured with a pair of counter-rotating RIMS 

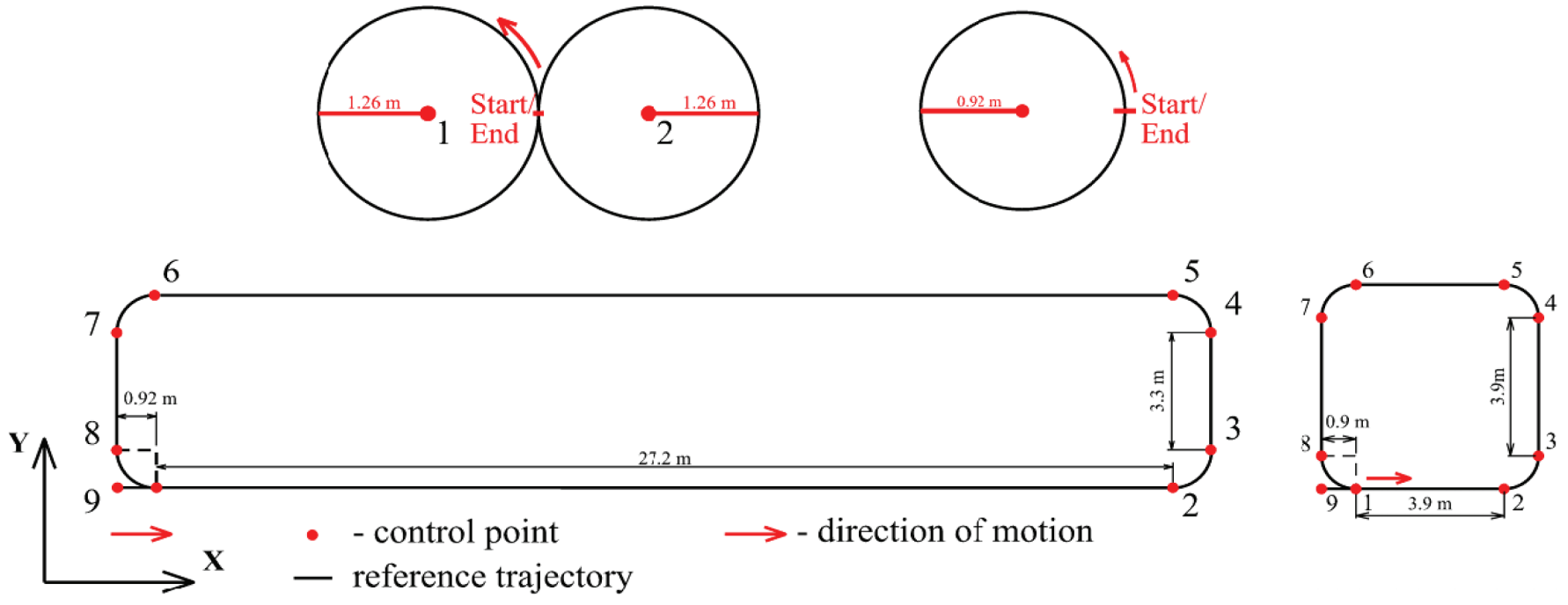

Fig. 14 Characteristics of the predefined trajectory for the second experiment

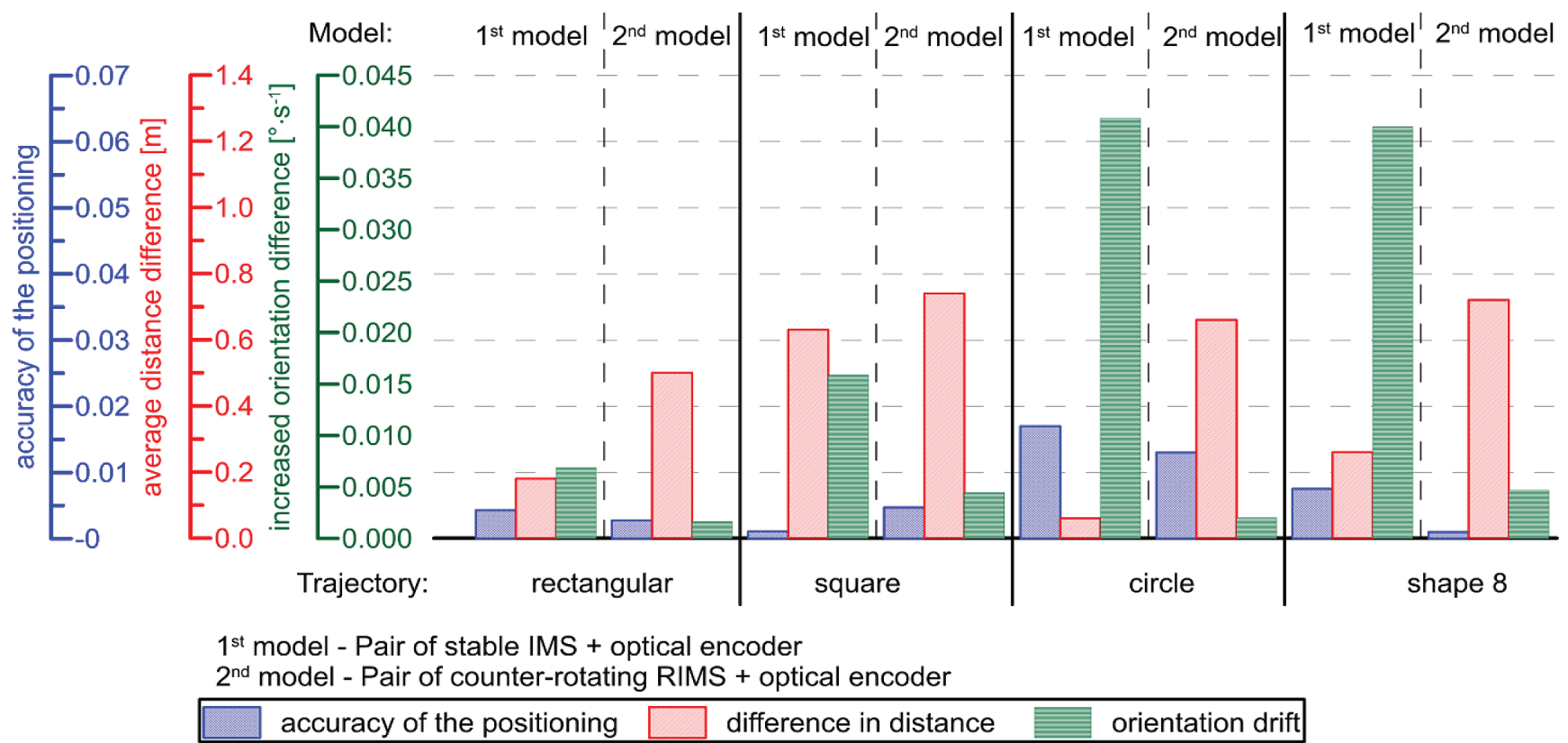

Fig. 15 Comparison between the proposed model using a pair of rotating counter RIMS with a model using a pair of stable IMS and an optical encoder

The efficiency of the proposed model measuring different trajectories (rectangular, square, circle and trajectory with an shape " 8 " Fig. 15), which represent motion under different dynamic conditions were analysed next. For each trajectory realized were measurements using a pair of stable (non-rotating) IMS and a pair of counter-rotating RIMS.

The results of the experiments (Fig. 15; Tab. 2; Tab. 3) show a significant improvement in the accuracy of the orientation in the case of the model using a pair of counter-rotating RIMS in all the measurements. This also leads to increased accuracy of the position. The comparison of the difference in the distance shows that the second model has a lower degree of accuracy in distance determining. As mentioned before, this problem is caused by changes in the angular velocity of the controlled rotation of RIMS, which have a negative effect on the filtration process of the centrifugal acceleration from the accelerations measured.

\section{CONCLUSION}

Thanks to the advantages of IMS (high frequency rate, independence a signal from external sources) compared to other navigation systems (GNSS, ultrasound), it has a significant role in monitoring the position of an object and its dynamic properties. The application of IMSs in geodesy are limited by the increasing position and orientation error, due to the accumulation of systematic errors of inertial sensors in the integration process.

This article brings a new approach to eliminating systematic errors of inertial sensors, based on the rotation of an IMS's platform. The controlled rotation of an IMS generates a periodic change in the IMS's signal, which leads to the periodic course of the DPSE. During the short period of the IMS rotation, the DSPE does not significantly change, which results to its periodic course and enables it elimination. With the increasing angular rate of the RIMS's rotation, the DPSE could be eliminated more efficiently, because the period of rotation 
Tab. 2 Results of the second experiment calculated using a pair of stable IMS and optical encoder

\begin{tabular}{l|c|c|c}
\hline Trajectory & $\begin{array}{c}\text { Accuracy of the } \\
\text { positioning }\end{array}$ & $\begin{array}{c}\text { Difference in } \\
\text { distance }[\mathrm{m}]\end{array}$ & $\begin{array}{c}\text { Orientation } \\
\text { Drift }\left[{ }^{\circ} \cdot \mathrm{s}^{-1}\right]\end{array}$ \\
\hline Rectangular & 0.004 & 0.18 & 0.0069 \\
\hline Square & 0.001 & 0.63 & 0.0159 \\
\hline Circle & 0.016 & 0.06 & 0.0408 \\
\hline Shape "8" & 0.007 & 0.26 & 0.0400 \\
\hline
\end{tabular}

is shorter. The next advantage of the higher angular velocity of the rotation is the better filtration of the centrifugal accelerations from the measured signal, because the frequency of the signal generated by the rotation is greater than the frequency generated by the IMS motion.

When using only one RIMS, it is not possible to eliminate systematic errors of inertial sensors, the sensitive axes of which are parallel to the axis of rotation of the RIMS. In order to solve this problem, an algorithm using a pair of counter-rotating RIMSs was designed. The principle of the proposed algorithm is based on the assumption that the same dynamic conditions of both RIMS (generated by the same angular rate of both RIMS) leads to the same course of the DPSE of the gyroscopes.

The results of the experimental measurements show the significant benefits of the proposed model for eliminating the DPSE of inertial sensors. RIMS is a suitable alternative to a combination of IMS with an optical encoder in cases, when it is not possible to use optical encoder. By using a pair of counter-rotating IMSs, the proposed data processing model effectively eliminates the DPSE of gyroscopes, which leads to increased precision in determined the orientation of a system's movement as well as increasing precision in the determining the position.
Tab. 3 Results of the second experiment calculate with using a pair of counter-rotating RIMS

\begin{tabular}{c|c|c|c}
\hline Trajectory & $\begin{array}{c}\text { Accuracy of } \\
\text { the positioning }\end{array}$ & $\begin{array}{c}\text { Difference in } \\
\text { distance }[\mathrm{m}]\end{array}$ & $\begin{array}{c}\text { Orientation } \\
\text { Drift }\left[{ }^{\circ} \cdot \mathrm{s}^{-1}\right]\end{array}$ \\
\hline Rectangular & 0.003 & 0.50 & 0.0016 \\
\hline Square & 0.005 & 0.74 & 0.0044 \\
\hline Circle & 0.013 & 0.66 & 0.0020 \\
\hline Shape "8" & 0.001 & 0.72 & 0.0046 \\
\hline
\end{tabular}

\section{Acknowledgement}

"This publication was supported by STU Foundation for Development of Talents - The Slavoj Family Scholarship."

\section{REFERENCES}

Bekkali, A. - Sanson, H. - Matsumoto, M. (2007). RFID indoor positioning based on probabilistic RFID map and Kalman filtering. In: Wireless and Mobile Computing, Networking and Communications WiMOB 2007. Third IEEE International Conference, pp. 21-21.

Ben, Y. (2011). Research on error modulating of SINS based on single-axis rotation. In: Complex Medical Engineering (CME), 2011 IEEE/ICME International Conference on. IEEE, 22.25. May 2011. China, pp. 293-297.

Collin, J. (2015). MEMS IMU Carouseling for Ground Vehicles. In: IEEE Transactions on Vehicular Technology, Vol. 64, No. 6, pp. 2242-2251.

Groves, P. D. (2008). GNSS Technology and Applications Series. Principles of GNSS, Inertial and Multisensor Integrated Navigation Systems. Artech House, London. 552 pp. ISBN-13:978-158053-255-6.

Kopáčik, A. - Kajánek, P. - Lipták, I. (2016). Systematic error elimination using additive measurements and combination of two low cost IMSs. In: IEEE Sensors Journal, Vol.16, No.16, pp. 6239-6248.

Ndjeng, A. (2011). Low cost IMU-Odometer-GPS ego localization for unusual maneuvers. Inf. Fusion, Vol. 12, No. 4, pp. 264-274.
Reimer, Ch. (2017). INS/GNSS Integration for Aerobatic Flight Applications and Aircraft Motion Surveying. In: Sensors, Vol. 17, No. 5, pp. 941-953.

Rizos, Ch. (2008). A hybrid system for navigation in GPS-challenged environments: case study. In: Proceedings, ION GNSS, Savannah, Georgia, pp. 16-19.

Sun, W. (2012). Accuracy improvement of SINS based on IMU rotational motion. In: IEEE Aerospace and Electronic Systems Magazine, Vol. 27, No. 8, pp. 1732-1740.

Wu, Y. (2014). Versatile land navigation using inertial sensors and odometry: Self-calibration, in-motion alignment and positioning. In: Proceedings of Inertial Sensors System Symposium ISS, pp. $1-19$.

Xu, Y. - Chen, X. - Li, Q. (2013). Autonomous integrated navigation for indoor robots utilizing on-line iterated extended rauchtung-striebel smoothing. In: Sensors, Vol. 12, No. 13, pp. 1593715953.

Yi. J. (2007). IMU-based localization and slip estimation for skidsteered mobile robots. In: Proceedings of International Conference on Intelligent Robots and Systems IROS, San Diego, pp. $2845-2850$. 